\title{
Percepción y programación deportiva en los estudiantes de la Universidad Estatal Península de Santa Elena
}

\section{Perception and sports programming in students of Santa Elena Peninsula State University}

\author{
Maritza Gisella Paula Chica ${ }^{1}$ \\ ${ }^{1}$ Universidad Estatal Península de Santa Elena, Ecuador \\ *m.g.pauli@gmail.com \\ DOI: https://doi.org/10.26871/killkanasocial.v4i2.732
}

\begin{abstract}
Resumen
El objetivo de esta investigación ha sido el análisis de la eficacia del rendimiento deportivo mediante un programa basado desde un diagnóstico previo en la asimilación de contenidos. Se ha utilizado un diseño casi-experimental de tipo pre-during programación. Un total de 82 estudiantes de la carrera de Educación Básica de la UPSE, han participado en este estudio desarrollado desde sus hogares en tiempos de pandemia. Utilizando 55 sesiones de entrenamientos. En una primera fase se evaluaron las adaptaciones de tiempos, repeticiones, pesos y movimientos visuales, posturales y técnicos del tiempo de respuesta a estímulos periféricos, la estereopsis y la atención selectiva focalizada durante la práctica deportiva. Los estudiantes y docente valoraron el rendimiento deportivo mediante los parámetros y estándares sobre tiempo, repeticiones, pesos y movimientos de concentración, velocidad perceptiva y visión periférica, que se concretan en los resultados diarios de cada wod de la programación. En una segunda fase de intervención se identifica el aumento progresivo de la carga según diagnóstico previo que permitía el ingreso de la programación desde la primera sesión. Repartidas de la siguiente manera: Sesiones adaptativas, sesiones con aumento de carga, sesiones con aumentos de tiempos y repeticiones, sesiones de restauración, sesiones de la continuidad del aumento progresivo. Adicionalmente, tanto los estudiantes como su entrenadora siguieron valorando cada sesión para el mencionado aumento progresivo de carga vista desde un macrociclo de entrenamiento. Se sigue dando continuidad a la programación con otros grupos más vulnerables; Los resultados obtenidos indican que el programa de entrenamiento proporciona mejoras estadísticamente significativas en la mayor parte de habilidades evaluadas (tiempos, repeticiones, pesos, movimientos visuales, posturales y técnicos), potenciando así mismo el rendimiento deportivo.
\end{abstract}

Palabras clave: atención selectiva, deportes, tiempos, repeticiones, pesos, movimientos visuales, posturales y técnicos.

\begin{abstract}
The objective of this research has been the analysis of the effectiveness of sports performance through a program, based on the assimilation of contents from a previous diagnosis. The almost-experimental design was applied like a pre-during program. Eighty-two students from Basic Education Career of UPSE have participated in this study, developed from their homes during pandemic period using 55 training sessions. In a first phase, the adaptations of times, repetitions, weights and visual, postural and technical movements of the response time to peripheral stimuli, teachers and students evaluated stereopsis and focused selective attention during sports practice that are specified in the daily results of each wod program. In a second phase of intervention, the progressive increase in the load is identified according to a previous diagnosis that allowed the entry of the programming from the first session. Distributed as follows: Adaptive sessions, sessions with increased load, and sessions with increases in time and repetitions, restoration sessions, sessions of the continuity of progressive increase. In addition, both students and their trainer continued evaluating each session for the mentioned progressive increase in load seen from a macro training cycle. The program continues with other more vulnerable groups. The results indicate that the training program provides statistically significant improvements in most of the evaluated skills (times, repetitions, weights, visual, postural and technical movements), thus enhancing sports performance.
\end{abstract}

Keywords: selective attention, sports, times, repetition, weights and visual movements, postures and technics.

\section{Introducción}

A nivel internacional se ha observado el acentuado interés hacia la estructura perceptivocognitiva en los deportis- tas dentro del contexto del rendimiento deportivo, así como la selección de la información, la atención, el proceso de la información, la parte técnica-táctica que resultan decisivos 
para el máximo rendimiento del deportista es importante identificar estas temáticas y ponerlas de manifiesto en el contexto nacional y/o su relación con parámetros que permitan pedir más adelante mediante normativas estándares.

Todos los deportes tienen diferencias entre grandes rasgos sean colectivos e individuales que le permite enfocar la capacidad visual dentro de la actividad deportiva. Esto dependerá de muchos factores externos con los que cuenta el deportista para su respectiva visión en la actividad.

Se ha tomado en cuenta que el deportista de élite depende de aquellas habilidades perceptivocognitivas como las capacidades físicas y motoras. (A. Williams, Grant, y Williams, 1999). La visión periférica, la estimación de la velocidad de desplazamiento, el tiempo de reacción visual entre otras múltiples acciones estarán implícitas en la parte perceptivocognitivas y que proporcionarán respuestas motoras adecuadas. (Erickson, 2007). Existen investigaciones enfocadas en Entrenamiento perceptivocognitivo con el Neurotracker 3D-MOT para potenciar el Rendimiento en tres modalidades deportivas los cuales sus resultados son satisfactorios y sus evidencias permiten enfocarlo hacia un laboratorio. El actual estudio se relaciona por primera ocasión con la literatura desde el área perceptivocognitivo, que permitirá más adelante enfocarlo hacia el laboratorio y así medir el entrenamiento y hacer un estudio comparativo con el Neurotracker 3D-MOT, con la investigación actual se difiere desde un trabajo de laboratorio hacia un trabajo más de campo tomando varias documentaciones teóricas investigativas acerca de lo perceptivocognitivo a nivel internacional.

La Percepción y programación deportiva dentro de este estudio, se la utiliza como variable independiente recayendo el cambio, la aportación, trasformación en la variable dependiente direccionado a los estudiantes de la Universidad Estatal Península de Santa Elena. Ante la imperiosa necesidad de aportar desde la práctica nuevos sistemas y/o elementos en el entrenamiento deportivo, con el fin de potenciar el rendimiento deportivo vía online en los estudiantes de la carrera de Educación Básica, hemos desarrollado el siguiente trabajo con el objetivo de determinar sí la percepción y programación deportiva influencia en los estudiantes de la Universidad Estatal Península de Santa Elena, en tiempo de pandemia covid-19 y, muy especialmente, si hay transferencia al rendimiento deportivo.

Numerosas investigaciones cuestionan si el entrenamiento y las habilidades visuales son transferidas hacia el rendimiento deportivo, se debería destacar el debate y ausencia de consensos entre los investigadores direccionados a estas áreas. Varios autores definen la potenciación de las habilidades visuales que van específicamente relacionadas con las diferentes modalidades deportivas y que éstas pueden tener a la vez un incremento en el rendimiento deportivo así lo manifiesta en sus obras académicas (Antúnez, 2003; Fradua, 1993; Quevedo y Solé, 1995; Vivas y Hellín, 2007), otros investigadores cuestionan la posibilidad de cualquier tipo de relación entre el entrenamiento visual y entrenamiento deportivo, así lo describen en sus investigaciones hacia deportista expertos y noveles, concluyendo en sus investigaciones que estas habilidades así como la visión periférica y función binocular, están totalmente diferenciadas entre los deportistas expertos, noveles y sedentarios, resaltando que dicha información receptada y captada es proceso del nivel cognitivo de cada individuo. (Abernethy, 1986; Garland y Barry, 1990; A. M. Williams, Davids, Burwitz, y Williams, 1994; A. Williams y cols., 1999).

Otro investigador (A. M. Williams, Burwitz, Davids, y Williams, 1992), denomina hardware visual a las habilidades pocos trabajadas en algunas disciplinas deportivas a la vez cuentan con instrumentos y técnicas optométricas, enfatizando en instrumentos y funciones estandarizadas incluyendo software que les permite tener un análisis en la selección, codificación, recuperación de ciertos datos visuales buscando estrategias para el mejoramiento de la atención, anticipación, memoria visual y visualización.

Desde el planteamiento de otros autores (Voss, Kramer, Prakash, Roberts, y Basak, 2009), describen a la evaluación de estas habilidades como el mecanismo cada vez más complicado el cual hace falta unificar esfuerzos para poder diseñar métodos científicos, objetivos válidos y fiables aportando a los modernos sistemas de Eye trackers.

\subsection{Importancia del Problema}

Tomando en cuenta algunas consideraciones se podría decir que existen algunas deficiencias de hardware en la aplicación de este tipo de investigación y que puede esto constituir una limitación en el rendimiento deportivo (Ferreira, 2002). Otros autores manifiestan con respecto a la utilización de software la distinción entre expertos, noveles. (Abernethy, 1986).

Estos investigadores adoctrinan que los deportistas son habilidosos para poder seleccionar y extraer información y llevarlas de forma relevante; organizándola, interpretándola de una manera mucho más rápida y eficaz, sustentando que esto aspectos cognitivos van a determinar las diferencias en el rendimiento deportivo y a la potencializado las que les interesan. (Ferreira, 2002).

Actualmente en la mejora del rendimiento deportivo, las técnicas desarrolladas en los deportes de alto nivel han llegado hacia la evolución en estos últimos años, llevándolo de forma sofisticadas en los entrenamientos; físico, tácticotécnico y psicológico. Sin embargo la función visual y perceptivocognitiva, todavía no han alcanzado las aportaciones requeridas de Antúnez, Argudo, Ruiz, Arias y García (2003), Adolphe, Vickers y Laplante (1997), o Williams y Grant (1999) que, mayoritariamente, utilizan paradigmas de oclusión espacial y temporal apoyados en tecnología audiovisual por lo cual nos deja la brecha para las próximas investigaciones.

Todas estas investigaciones van marcando totalmente la diferencia entre varios autores que se encuentran citados anteriormente (Garland y Barry, 1990; Ferreira, 2002; Abernethy, 1986), donde defienden que las habilidades 
pertenecientes al software visual proporcionan al atleta una importante ventaja respecto a los otros.

Antes la imperiosa necesidad de aportar en los nuevos avances del entrenamiento deportivo y a la vez con el fin de potenciar el rendimiento deportivo, hemos decidido desarrollar el siguiente trabajo con el objetivo de determinar si el entrenamiento de las habilidades perceptivocognitivas con la programación deportiva, influencia la funcionabilidad del sistema visual, atención selectiva, y transferencia, al rendimiento deportivo.

\section{Metodología}

En el presente trabajo de investigación, se utiliza un diseño cuasi-experimental pre-during vista desde 2 fases. La efectividad del programa del entrenamiento ha sido valorada a partir de las comparaciones de los resultados en cada uno de los wod empleados y a la vez mediante un diagnóstico antes de empezar la actividad, el cuál ha permitido el análisis sobre tiempo y efectividad del programa del entrenamiento valorado diariamente. Cada estudiante ha sido capaz de identificar; sesiones adaptativas, sesiones con aumento de carga, sesiones con aumentos de tiempos y repeticiones, sesiones de restauración, sesiones de la continuidad del aumento progresivo durante la práctica deportiva. Los datos se encuentran registrados en el libro de memorias con el fin de identificar la evolución del registro histórico empleado en cada wod. Así la variable Independiente recae en La percepción y programación y la variable dependiente estuvo enfoca en los estudiantes. Valoramos que la muestra alcanzada fue de total de 82 estudiantes que pertenecen a la Facultad de Ciencias de la Educación e Idiomas, la cual participaron en la investigación; 35 varones y 40 mujeres, todas las actividades las llevaron a cabo dentro de casa en tiempos de Pandemia Covid-19, buscando materiales que se encontraban a su alrededor para lograr el objetivo alcanzado en cada uno de los wod. Del $100 \%$ del estudiantado se identificó que existía el $3 \%$ que realizaban actividad física, por lo tanto la influencia de los entrenamientos se veía muy marcada y a la vez diferenciada en los movimientos, tiempos repeticiones, restauración en cada uno de los ejercicios, sin embargo el $90 \%$ de estudiantes desconocían movimientos, tiempos, repeticiones, aumento progresivo, sesiones de restauración por lo que se marcaba y afectaba en los distintos aspectos de la preparación deportiva (físico, técnica-táctica y psicológico). Esto apoyaría las aportaciones de los autores anteriormente mencionados desde la teoría y metodología (Bompa, 1999); así como en el control de factores y problemas del entrenamiento enfocados en los fundamentos científicos (Brogli, 1991; Costes, 1972; Diachkov, 1961).

La esencia de la programación del entrenamiento deportivo, visto desde los métodos de entrenamiento deportivo, ha permitido el aumento progresivo de la carga. Así se manifiesta en la validación por expertos los cuales los doctores participantes contestaron un cuestionario en línea conformado por diez preguntas, tomando a la vez una muestra aleatoria de los wod y de los resultados. Buscando la finalidad de obtener elementos que permitan la experiencia de validar el contenido de instrumentos, recogimos las opiniones de académicos que han participado en varias investigaciones desde el entrenamiento deportivo. Los datos se obtuvieron a través de un cuestionario aplicado vía online a distintos académicos; los participantes fueron diez doctores con título de entrenamiento deportivo pertenecientes a cuatro instituciones de educación superior: Universidad Autónoma de Nuevo León, México, Universidad Nacional de Lomas de Zamora, Universidad de Cultura Física Manuel Fajardo; su antigüedad laboral oscila entre dieciséis y treinta y un años. La experiencia laboral de los doctores se enfoca en investigaciones y docencia universitaria, y su formación se centra en áreas como investigación en el rendimiento deportivo, educación superior, tecnología deportiva.

En el libro de memoria se puede identificar el logro de los máximos resultados deportivos determinados por la creciente funcional valoradas desde los resultados y estímulos del ambiente natural del individuo hacia una manifestación máxima de sus capacidades motrices, motoras evolutivas, Intelectuales entre otras, alcanzadas en el proceso de formación de la programación.

Material e instalaciones; En la realización de la exploración de movimientos visuales, posturales y técnicos quedan como respuesta a los estímulos periféricos en donde los estudiante fueron capaces de utilizar diferentes implemento que le permitieron adaptarlos en cada una de los ejercicios empleados, Baldes, palo de escobas, maletas, libros, mancuernas, pesas caseras, Llantas pequeñas hasta llantas de camión, entre otros materiales caseros. En los link de youtube se pueden observar los vídeos en donde se describe cada uno de los ejercicios, implementos, adaptaciones y resultados diarios.

Para engarzar los videos se empleó el libro de memorias el cual se encuentra en la página de moodle de la $\mathrm{Ca}$ rrera de Educación Básica, asignatura Educación Física. En el libro de memoria se puede observar el carácter variado del entrenamiento deportivo en la utilización de medios que permiten influir sobre el organismo de nuestros estudiantes, a la vez, la diversidad ha permitido buscar y desarrollar el carácter de la disciplina en la práctica deportiva.

Los medios que se utilizaron se enfocan específicamente en ejercicio de preparación general desde su inicio dando énfasis al ejercicio de preparación especial. Este impone emplear el entrenamiento desde el macro, meso y micro llevada hacia la unidad de entrenamiento.

El entrenamiento y mejora se ven implícitas desde la función; macro meso, micro y unidad de entrenamiento, la misma que ha sido patentada por otros autores desde los problemas principales de la teoría de los Sistemas funcionales (Anojin, 1973; Bachvarov, 1982); buscando la diversidad del entrenamiento, sin embargo (Bernstein, 1947) sustenta la Formación de los Movimientos, (Di Prampero, 
1988) describe los aspectos energéticos del desempeño humano.

Por otra parte el programa informático utilizado es Microsoft excel 2010, la cual nos permitió identificar desde los por cientos, vistos en cada macrocilo, mesociclo y microciclos descritos en cada wod de entrenamiento. (Ver gráfico 5). La visualización de videos un día antes sirvió para la valoración de la capacidad de respuesta a estímulo periféricos involucrando a la vez la tarea central del control visual, lo que le permite al estudiantado visualizarlos y ejecutarlos el día de la actividad. La totalidad de los ejercicios fueron hechos desde casa. Con respecto al control del rendimiento, operativizado en las variables de concentración visual y visión periférica, esto nos permitirá más adelante utilizarlas desde un laboratorio clínico involucrando una tarea central del control visual como lo describe (Quevedo, Cardona, Solé, y Bach, 2001).

Procedimientos; Antes de empezar la programación del entrenamiento se tomó en cuenta cada una de las actividades; repeticiones, tiempos, nomenclatura de ejercicios por la cual ellos iban atravesar durante las 55 sesiones. El carácter discutible de las 55 sesiones que se llevaron en conjunto con los objetivos planteados direccionados al deporte así como las exigencias morfo funcional del organismo. La especificidad de la estructura de la actividad; La forma de garantizar la parte técnica- material y financiera durante toda la práctica del entrenamiento. Se abordó las concepciones teóricas metodológicas y experiencia que deben de llevar en la práctica de la actividad física.

Se identificó algunas nociones primarias fragmentadas sobre una estructura en el contenido del proceso de entrenamiento e influencia de factores exógenos entre "pandemia y el papel conductor de la preparación física general durante el tiempo de confinamiento. Estas grandes teorías conductuales de la preparación física son abordadas a la vez por (Marshall y Saffer, 1908).

La Periodización del proceso de entrenamiento, va direccionada a la esencia de los cambios periódicos que se producen dentro de la estructura y su contenido bajo la influencia del tiempo de las cargas de entrenamiento. La esencia de la periodización de la programación permitirá producir una estructura y contenido con sus componentes; partes, tipos provocando una estructura básica de proceso de entrenamiento. Estamos en presencia de una estructura básica uniciclica tomando en cuenta el modelo de la preparación del deportista de élite de remo de Bulgaria durante 1996/1997 (Neykov, 1996).

Se tomó en cuenta varios aspecto con relación a la meso estructura, del proceso de entrenamiento en donde se encuentran aspectos más concreto en los diferentes mesosciclo que caracterizan las ondas medias del proceso adaptativo en el tiempo, peso y repeticiones. En el proceso de investigación se identifica mesociclos de distinta duración de contenido. Algunos de estos mesociclos se tomarán en cuenta en el proceso de la aplicación del programa de entrenamiento deportivo en los estudiantes de la carrera de educación básica; mesociclo básico, mesociclo estabilizador, mesociclo precompetitivo y mesociclo de descarga. En el programa se puede identificar una microestructura del proceso de entrenamiento vista desde las sesiones de entrenamiento diarias enfocadas desde la magnitud de la carga así como la estructura de los microciclos de entrenamiento entrelazadas desde la unidad básica estructural que vincula todas las actividades en una unidad orgánica y que representa un fragmento en todo el proceso docente educativo, tiene un promedio de duración entre 4 a 10 días. La metodología en la confección de los microciclos de entrenamiento permitió las regularidades objetivas de proceso adaptativo, gasto, recuperación, superrecuperación y regreso a la posición inicial.

Cuestiones metodológicas generales dentro del control del proceso de entrenamiento permitió medir los resultados descriptos en el libro de memorias y en los videos subidos en sus cuentas de YouTube, como parte de la evidencia del programa deportivo. El control y regulación de la preparación deportiva cuenta con parámetros mensurables visto desde los tres niveles; integrales, complejos y diferenciales. Nos enfocamos en tres formas básicas de control en el proceso de entrenamiento moderno; control operativo, control corriente, control de tapas. En el control operativo observamos la actuación inmediata del organismo en relación a la reacción del proceso de la actividad de entrenamiento a partir de aquí se emplearon una serie de metodologías aplicadas en las condiciones naturales desde casa. A pesar de este esfuerzo en lograrse sistematizar los conocimientos teóricos y metodológicos la experiencia práctica ha permitido aclarar las características básicas del control del gran deporte (Fleishman, 1965; Zatsiorsky, Y, y P, 1969; Celicovsky, 1972; Grosser, 1972).

Es importante mencionar que la progresión del control de la carga depende de la habilidad del entrenador para que sus atletas puedan optimizar las cargas de entrenamiento. La complejidad de esta problemática está condicionada con el número de la variante de la carga externa. Al tener en cuenta la carga que se emite; complejo, dinámico y autorregulador. Se hace necesario dentro de la programación realizar la respectiva clasificación de las influencias del entrenamiento por magnitud, carácter y orientación. En el libro de memoria se puede observar el índice de la carga funcional en donde el índice de las reacciones momentáneas del organismo se controlan mediante la carga interna (funcional); músculos esqueléticos, los órganos de la circulación sanguínea y la respiración, podríamos decir que estos grandes cambios están relacionados con grandes pérdidas energéticas.

En cada semana se observa una sesión diaria con tiempo de duración 45 minutos hasta 50 minutos, el programa de entrenamiento duro 16 semanas compuestas en 55 sesiones. Las evaluaciones eran continuas antes durante y después de cada entrenamiento, el mismo que permitía medir en tiempo, repeticiones y pesos. 


\section{Resultados}

\subsection{Diagnóstico de los Resultados}

Se aplicó la encuesta a estudiantes de la carrera educación básica de la signatura educación física; con esta información se trata de inferir en las acciones generadas por la perfección y programación deportiva en los estudiante de la Facultad de Ciencias de la Educación e Idiomas de la Universidad Estatal Península Santa Elena.

Entre los resultados más representativos se destaca que la función del sistema nervioso sobre la acción de observar vídeos un día antes de ejercicios, el $100 \%$ ha contestado que coordina las funciones tanto conscientes como inconscientes.

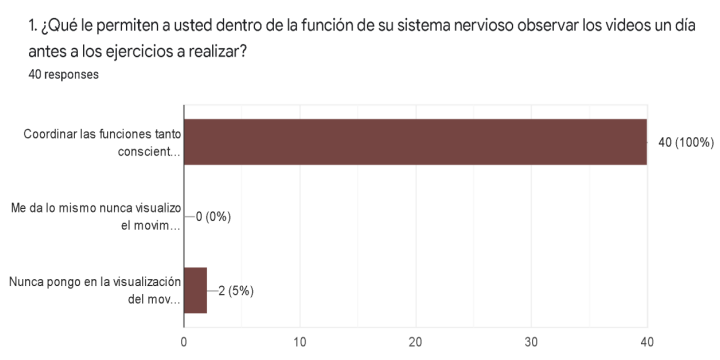

Figura 1: Función de Sistema Nervioso visualizando videos un día antes de los ejercicios

Fuente: Paula Maritza, Ecuador, 2020

Consultándoles sobre la función del sistema nervioso central en la inhalación y la exhalación el 75,6\% pone de manifiesto durante el ejercicio, sin embargo el $19,5 \%$ inhala y exhala después del ejercicio, sin embargo el $4 \%$ manifiesta que lo realiza antes del ejercicio. En este escenario de dudas y respuestas se destaca a La inhalación y exhalación durante el ejercicio físico.

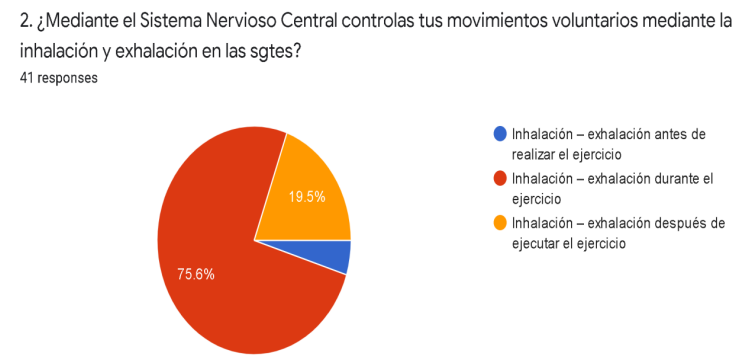

Figura 2: Función de Sistema Nervioso Central en la inhalación y exhalación

Fuente: Paula Maritza, Ecuador, 2020

Preguntando acerca del dominio o conocimiento de las neuronas espejo en la mejora del rendimiento deportivo se identifica qué el $95,1 \%$ si domina este conocimiento, sin embargo el 4,9\% desconoce acerca de la práctica de visualización que lleva la función las neuronas de espejo.
7. Uno de los descubrimientos más importantes en la neurociencia en el deporte como son las neuronas espejo, cree usted que le permite un mejor rendimiento de la práctica de la actividad fisica? 41 responses
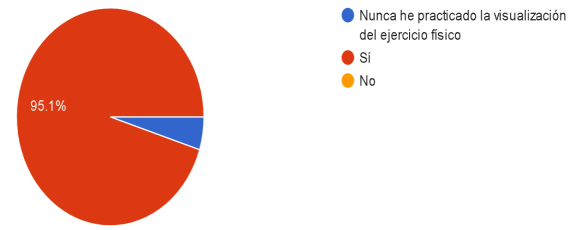

Figura 3: Neuronas espejos mejora en el rendimiento deportivo

$$
\text { Fuente: Paula Maritza, Ecuador, } 2020
$$

Se les realizó cuestionamientos sobre cómo se adapta la respuesta de estímulos periféricos en la programación semanal, aquí se observa que el $100 \%$ afirma que la respuesta a los estímulos periféricos, lo encontraron durante la programación en tiempo, repeticiones, pesos y movimientos visuales posturales y técnica. Cabe resaltar que era pertinente la búsqueda de estímulos periféricos mediante la programación debido a que la misma se realiza sobre tiempos, repeticiones, movimientos visuales, posturales y técnicos.

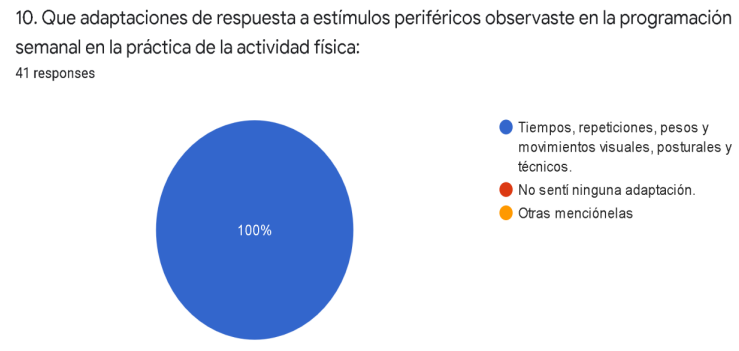

Figura 4: Adaptaciones a los estímulos periféricos durante la programación

Fuente: Paula Maritza, Ecuador, 2020

Dentro de la estructura de la carga, se ha observado el desarrollo de la teoría y práctica del deporte vista desde una compleja estructura funcional de componentes que constantemente van cambiando sobre la marcha en la preparación del deportista. Esta gráfica identifica la correlación óptima entre los componentes de un mesociclo en el entrenamiento deportivo, enfocado en el contenido básico de la labor del entrenador. En esta gráfica se observa la magnitud de la carga funcional y su dosificación durante el proceso de entrenamiento. El perfeccionamiento de las capacidades funcionales del organismo permite en esta gráfica identificar el incremento de esa carga externa, en la Magnitud, el carácter de orientación en esa carga externa. Dentro del volumen cuantitativo, se observa el trabajo efectuado en una sesión durante un determinado periodo de tiempo, La intensidad, se intensifica mediante el grado de los esfuerzos en cada uno de los ejercicios; es decir la cantidad de repeticiones sobre un tiempo determinado. Corroborado desde la teoría por varios autores vista desde la periodización del 
entrenamiento deportivo. (Matveyev, 1965; Platonov, 1998; Volkov, A, y M, 1990; Zhaliazkov, 1986)



Figura 5: Programación vista desde el comportamiento individualizado de las cargas

Fuente: Paula Maritza, Ecuador, 2020

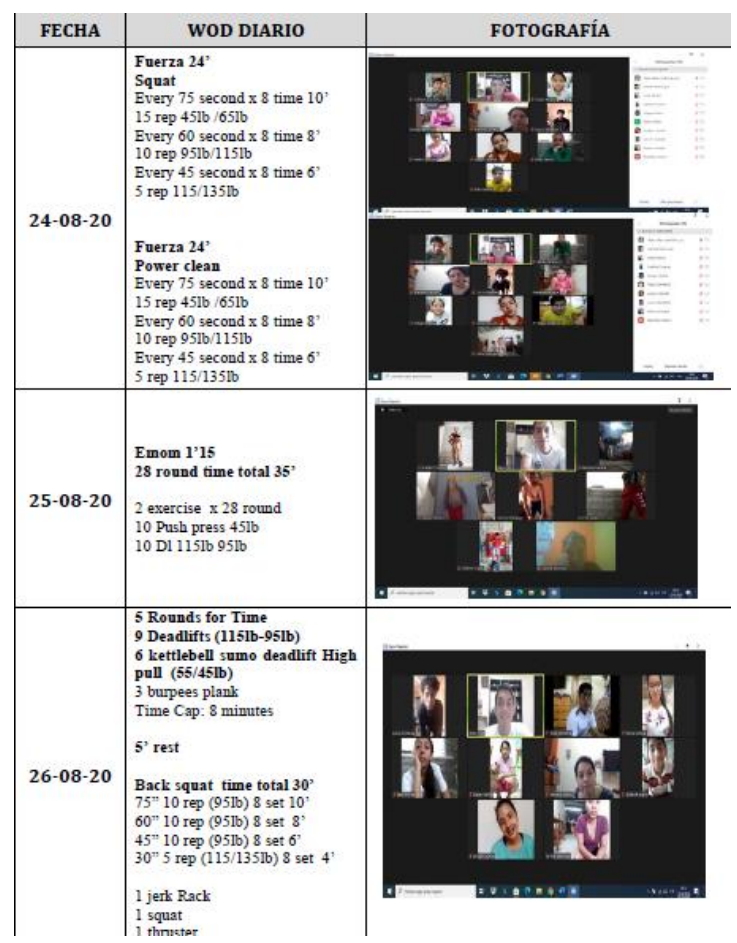

Figura 6: Programación vista desde el acondicionamiento metabólico (metcom) diario

Fuente: Paula Maritza, Ecuador, 2020

Dando respuesta a la programación vista de las sesiones con aumento de carga, sesiones con aumentos de tiempos y repeticiones, sesiones de restauración, sesiones de la continuidad del aumento progresivo, se puede observar componentes de la carga externa y efecto funcional que van en dependencia sinónima debido a las distintas posibilidades adaptativas de cada sistema para asumir y transformar los movimientos visto sobre tiempo, espacio y repeticiones, el efecto funcional de los ejercicios practicados y observarlos ejercicios menos practicados permiten distinguir el proceso continuo de la carga. Se puede observar las distintas funciones metabólicas y las morfoestructuras vistas desde el macro, meso y micro en función de la unidad de entrenamiento. Así también lo confirma en sus obras escritas (Sarsania, 1991). Se puede observar la carga con influencia primordial aláctica, así como la carga con influencia anaeróbica glicolítico, carga con influencia mixta, aerobio-anaerobio, carga con influencia aerobia; carga con influencia anabólica. Las sistematización de las cargas de entrenamiento permiten elevar el promedio de estadísticas de su magnitud, carácter y orientación.

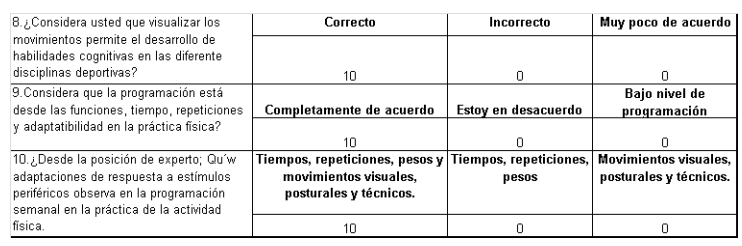

Figura 7: Selección de preguntas Validada por expertos Fuente: Paula Maritza, Ecuador, 2020

\section{Discusión}

Otros autores sustentan el sentido somato sensorial para el funcionamiento motor suave en la propiocepción llamado (sentido del movimiento). Describiendo que los propioceptores se encuentran en los músculos así como las articulaciones y que ellos detectan las posiciones los movimientos, la fuerza de las extremidades (Proske U, 2012). Recordemos que la aferencia propioceptiva que va al cerebro se integra de forma única al sistema vestibular para luego proporcionar al cerebro la información acerca del estado interno del sistema locomotor y su debida orientación espacial.

Se evidencia mediante la publicación en diferentes revistas científicas acerca de bibliografía especializadas (Adolphe y cols., 1997; Singer N y cols., 1994). La mismas que evalúan y contrastan las diferencias de las habilidades persceptivocognitivas en donde se encuentra pocos trabajos que se indague aquellos efectos de estos tipos de entrenamiento en la mejora del rendimiento deportivo (Antúnez, 2003; Farrow y Abernethy, 2002). A la vez se estudian capacidades así como aquellos métodos muy diversos que facilita la contextualización y establecimiento las relaciones con los resultados en nuestro programa. Por este motivo la discusión se basa entrando básicamente en los datos obtenidos durante la presente investigación.

Habilidad visual se han observado en este estudio que permite identificar que casi todas las habilidades han ido en el aumento progresivo y especialmente significativo en lo que corresponde a los movimientos. Esto permite el apoyo de aquellas aportaciones de varios autores como: (Antúnez, 2003; Fradua, 1993; Quevedo y Solé, 1995; Vivas y Hellín, 2007). Estos autores defienden el mejoramiento de las habilidades visuales mediante el entrenamiento deportivo lo que la vez permite integrar elementos conjuntos con acciones propias, vistas desde cada deporte. La programación del entrenamiento deportivo vista en este trabajo radica en el aumento progresivo de la carga descrito desde un macro, 
meso y micro y unidad de entrenamiento. En dónde se observa al entrenamiento como proceso de regulación de la carga aportando en la mejora de las habilidades visual y física.

Otros autores (Lasky y Lasky, 1990; McKee y Westheimer, 1978; Quevedo y Solé, 1995). han visto la perfección potenciada de manera estadística, en la demostración de esta habilidad visual en la que mejora mediante la técnica de entrenamiento. Posiblemente la toma de laboratorio podría replicarse en este estudio u en otros el cuál permita medir la sensibilidad de los contrastes que se puedan observar de manera significativa a la frecuencia parcial así como lo manifiesta (Kluka, Love, Sanet, Hillier, y Schneider, 1995).

Varios autores (Long y Riggs, 1991; Fradua, 1993) constatan mediante investigaciones que tanto la visión periférica, así como las habilidad visuales mejoran según los programa de entrenamiento que se ponga de manifiesto durante la práctica de la actividad física. Es sorprendente que la visión periférica y las respuestas a estímulos periféricos son habilidades esenciales durante el entrenamiento deportivo (Erickson, 2007), sin embargo clínicamente son significativas, en esta investigación hemos identificado durante la practica la mejora de las mismas constatadas mediante instrumentos empíricos sin contar con un laboratorio. Durante el proceso investigativo se pretende valorar en los próximos trabajos científicos mediante un laboratorio y dar respuestas a las nuevas interrogantes planteadas.

Durante el proceso de investigación se pudo corroborar que ha existido la mejora de forma estadística significativa con el presente programa de entrenamiento en cuanto a la atención selectiva. La misma que se observaba una gran demanda en atención selectiva focalizada y dividida durante el entrenamiento.

Varios autores como (Hagemann, Strauss, y CañalBruland, 2006). Según (Mann, Williams, Ward, y Janelle, 2007) identifican la mejora de las Variables atencionales como parte crucial para el éxito en el desarrollo de la práctica de la actividad física.

Rendimiento deportivo valorado mediante las variables concentración visual, velocidad perceptiva y conciencia periférica: Varios investigadores han realizado estudios clínicos mediante cuestionarios de escalas visuales analógicas que valoraba las tres variables mencionadas obteniendo como resultado la mejora progresiva en las tres variables que evalúan mediante el rendimiento deportivo Lluïsa Quevedo Junyent, Anna Padrós Blázquez, Joan Solé i Fortó, Genís Cardona Torradeflotot.

Se destaca que dentro de la semana 8 y 16 del cronograma académico de la UPSE, se observó una ligera baja de los registros de todas las habilidades adquiridas durante el tiempo de preparación de los estudiantes debido a que se manifestó la parada en la semana 8 y 16 de exámenes parciales de cada ciclo, que rompió la respectiva continuidad del programa de entrenamiento. Además podemos identificar la disminución momentánea de la motivación y frustración que pudieron alcanzar en el empeoramiento de las habilidades adquiridas sufridas al cambiar el protocolo y pasar de hacer el entrenamiento continuo a una semana 8 y 16 de parada de la programación debido a los exámenes parciales.

Se identifica claramente que en la parada de la programación ha implicado la disminución en la mejora de sus movimientos y esto permite tomar nuevas medidas más adelante buscando hacer una duración mínima para garantizar las mejoras y de esta manera no perder el aumento progresivo de la carga. Al examinar el entrenamiento deportivo visto desde el proceso adaptativo desde el punto de vista del enfoque sistemático es importante señalar que dicho concepto comprende tanto las reacciones adaptativas directas del organismo como aquellos resultados del proceso adaptativo. Desde la dimensión inmediata dinámica y acumulativa estable. Estas relaciones mutuas de diferente eslabones las manifiesta (Meerson, 1981). Permitiendo llevar sesiones de entrenamiento esporádicas de refuerzo para mantener los niveles de rendimiento óptimo. Todo esto reafirmaría las conclusiones vista por varios autores como (Antúnez, 2003; Quevedo y Solé, 1995), con jugadores de balonmano y tiradores/ lanzadores expertos, respectivamente.

\section{Conclusión}

Podríamos concluir que la percepción y programación deportiva en los estudiantes de la Universidad Estatal Península de Santa Elena, se ha llevado a cabo en tiempo de Confinamiento y ha permitido la mejora de las habilidades de la visión periférica. Adicionalmente podríamos decir que la atención selectiva enfocada a los ejercicios ha mejorado en sus técnicas, tiempos, repeticiones.

A nivel global podríamos estar diciendo que se constata dentro del programa de entrenamiento evidenciado en un libro de memorias, el fortalecimiento del programa de entrenamiento deportivo operativizado en las variables de concentración; visual, velocidad perceptiva y la conciencia periférica, durante la actividad deportiva llevada desde casa en tiempo de pandemia covid-19, durante 55 sesiones, vistas desde 16 semanas, una hora diaria, de lunes a viernes.

Finalmente, y siempre con la debida prudencia, podríamos generalizar que el entrenamiento de las habilidades perceptivocognitivas con este programa de entrenamiento deportivo vía online o similares podrían potenciar el rendimiento deportivo en otras disciplinas deportivas, como por ejemplo en los deportes individuales, atletismo, levantamiento de pesas, así como en otros ámbitos que permitan incluir situaciones donde se requiere de reacciones rápidas en entornos virtuales; por ejemplo, El tiempo; podríamos decir que este tipo de entrenamiento sería un supuesto resultado muy útil para los deportistas ya que les permite medirse sobre el tiempo visual en línea con el objetivo de conservar su parte psicológica activa y a la vez motivados, tomando en consideración que un entrenamiento siempre 
estará presente un reto de mejora continua, implicada de manera directa en la rutina del deportista.

\section{Referencias Bibliográficas}

Abernethy, B. (1986). Enhancing sports performance through clinical and experimental optometry. Clinical and Experimental Optometry. doi:10.1111.

Adolphe, R., Vickers, J., y Laplante, G. (1997). The effects of training.

Anojin, P. (1973). Problemas principales de la Teoría General de los Sistemas Funcionales. Principios de la Organización Sistemática de las Funciones). Moscú.

Antúnez, A. (2003). La interpretación en la portera de balonmano. Efectos de un programa de entrenamiento perceptivo-motriz. Murcia: (Tesis doctoral). Universidad de Murcia.

Bachvarov, M. (1982). Diversidad del entrenamiento Deportivo. Meditzina y Fiscultura (Medicina y Educación Física). Sofía.

Bernstein, N. (1947). Sobre la formación d elos movimientos . Moscú: medguiz (editorial Estatal de Medicina.

Bompa, T. (1999). Periodization, Theory and Metodology of training. Moscú: 4 edicion.

Brogli, Y. (1991). Control de los Factores del Resultado Deportivo. Cuestiones de Educación Física, $N 8$. Sofía.

Celicovsky, S. (1972). Antropomotorika. Teorie Telesnych Cvicent. 1972: SPN Praha.

Costes, N. (1972). Interval Training. Mountain View. Worls Publ.

Di Prampero, P. (1988). Aspects Énergétiiques de la Performance Humaine. Paris: M. Rien.

Diachkov, V. (1961). Problemas del Entrenamiento Deportivo. Moscú: FyS.

Erickson, G. B. (2007). Sport Vision. Vision care for the enhancemen of sports performance. Filadelfia: Butterworth \& Heinemann.

Farrow, D., y Abernethy, B. (2002). Can anticipatory skills be learned through implicit video-based perceptual training? Journal of Sports Sciences, (20)6, 471485.

Ferreira, J. T. (2002). Sports Vision as a Hardware and Software system. Eyesite.

Fleishman, E. (1965). The Structure and Measurement of Physical Fitness. Prentice -Hall.

Fradua, J. L. (1993). Efectos del entrenamiento de la visión periférica en el rendimiento del futbolista. Granada: Universidad de Granada.

Garland, D. J., y Barry, J. R. (1990). Sports expertise: The cognitive advantage. Perceptual Motor Skills.

Grosser, M. (1972). Einfuhrung in die theorie Sportmotorischen Test. Turner Sport.

Hagemann, N., Strauss, B., y Cañal-Bruland, R. (2006). Training Perceptual Skill by Orienting Visual Attention. Journal of Sport \& Exercise Psychology.
Kluka, D. A., Love, P., Sanet, R. B., Hillier, C., y Schneider, H. (1995). Contrast Sensivity function profiling: By sport and sport ability level. International Journal of Sports Vision.

Lasky, D. I., y Lasky, A. M. (1990). Stereoscopic eye exercises and visual acuity. Perceptual and Motor Skills.

Long, G. M., y Riggs, C. A. (1991). Training effects on dynamic visual acuity with free-head viewing. Perception.

Mann, D. T., Williams, A. M., Ward, P., y Janelle, C. M. (2007). Perceptual-cognitive expertise in sport: A meta-analisis. Journal of Sport \& Exercice Psycho$\log y$.

Marshall, J., y Saffer, E. (1908). No Title.

Matveyev, L. (1965). El probelma de la periodización del Entrenamiento Deportivo. Moscú: Educación Física y Deporte.

McKee, S., y Westheimer, G. (1978). Improvement in vernier acuity with practice. Perception and Psychophysics.

Meerson, f. (1981). Adpatación, Estrés y Profiláctica. Moscú: Nauka (Ciencia).

Platonov, V. (1998). Sobre el Çoncepto de Periodización del Entrenamiento Deportivo z el Desarrollo de la teoría General de la Preparación de los deportistas. Moscú: teoría y Práctica de la Educación Física.

Proske U, G. S. C. (2012). The proprioceptive senses: their roles in signaling body shape, body position and movement, and muscle force. Physiol Rev. 92(4), 1651-1697.

Quevedo, L., Cardona, G., Solé, J., y Bach, E. (2001). Perfil Visual y auditivo de los árbitros de Primera división de la Liga Española de Fútbol. Ver y Oír.

Quevedo, L., y Solé, J. (1995). Visual training programme applied to precisión shooting. Ophthalmic and Physiological Optics.

Sarsania, S. (1991). La Preparación Física en los Juegos Deportivos. Moscú.

Singer N, R., Cauraugh, J. H., Chen, D., M., G., Frehlich, S. G., y Wang, L. (1994). Training mental quickness in beginning/intermédiate tennis player. The Sports Psychologist, (8) 305 -318.

Vivas, X., y Hellín, A. (2007). Intervención optométrica en el hockeysobre patines. Apunts. Educación Física y Deportes.

Volkov, N., A, K., y M, H. (1990). Teoría y Práctica del entrenamiento Interválico en el Deporte. Moscú.

Voss, M., Kramer, A. F., Prakash, R. S., Roberts, B., y Basak, C. (2009). Are expert athletes "expert" in the cognitive laboratory? A meta-analytic review of cognition and sport expertise. Applied Cognitive Psychology.

Williams, A., Grant, A., y Williams, J. (1999). Visual perception and action in sport. New York: Routledge. New York: Routledge. 
Williams, A. M., Burwitz, L., Davids, K., y Williams, J. G. (1992). Perception and Action in Sport. Journal of Human Movement Studies.

Williams, A. M., Davids, K., Burwitz, L., y Williams, J. G. (1994). Visual search strategies of experienced and inexperienced soccer players. Research Quarterly for Sport and Exercise.

Zatsiorsky, T., Y, G., y P, N. (1969). Cibernética, Matemáticas, Deporte. Moscú: Educación Fisica y Deporte.

Zhaliazkov, T. (1986). Teoría y Metodología del Entrenamiento Deportivo . Medicina y Educación Física, Sofía.

Recibido: 10 de diciembre de 2019

Aceptado: 26 de abril de 2020

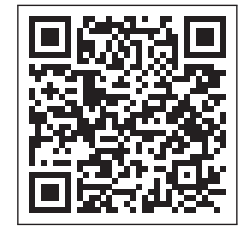


